\title{
Voice Pitch - A Valid Indicator of One's Unfaithfulness in Committed Relationships?
}

\author{
Christoph Schild $^{1,2}$ (1) Julia Stern ${ }^{3} \cdot$ Lars Penke $^{3} \cdot$ Ingo Zettler $^{1} \cdot$
}

Received: 29 October 2019 / Revised: 19 August 2020 / Accepted: 1 October 2020 /

Published online: 16 October 2020

(C) The Author(s) 2020

\begin{abstract}
Objectives When judging a male speakers' likelihood to act sexually unfaithful in a committed relationship, listeners rely on the speakers' voice pitch such that lower voice pitch is perceived as indicating being more unfaithful. In line with this finding, a recent study (Schild et al. Behavioral Ecology, 2020) provided first evidence that voice pitch might indeed be a valid cue to sexual infidelity in men. In this study, male speakers with lower voice pitch, as indicated by lower mean fundamental frequency (mean F0), were actually more likely to report having been sexually unfaithful in the past. Although these results fit the literature on vocal perceptions in contexts of sexual selection, the study was, as stated by the authors, underpowered. Further, the study solely focused on male speakers, which leaves it open whether these findings are also transferable to female speakers.
\end{abstract}

Methods We reanalyzed three datasets (Asendorpf et al. European Journal of Personality, 25, 16-30, 2011; Penke and Asendorpf Journal of Personality and Social Psychology, 95, 1113-1135, 2008; Stern et al. 2020) that include voice recordings and infidelity data of overall 865 individuals $(63,36 \%$ female) in order to test the replicability of and further extend past research.

Results A significant negative link between mean F0 and self-reported infidelity was found in only one out of two datasets for men and only one out of three datasets for women. Two meta-analyses (accounting for the sample sizes and including data of Schild et al. 2020), however, suggest that lower mean F0 might be a valid indicator of higher probability of self-reported infidelity in both men and women.

Conclusions In line with prior research, higher masculinity, as indicated by lower mean F0, seems to be linked to self-reported infidelity in both men and women. However, given methodological shortcomings, future studies should set out to further delve into these findings.

Keywords Voice pitch · Infidelity · Fundamental frequency $\cdot$ Trustworthiness

Christoph Schild

christoph.schild@uni-siegen.de

Extended author information available on the last page of the article 


\section{Theoretical Background}

The human voice has been found to play a crucial role in contexts of sexual selection (Pisanski et al. 2018; Puts et al. 2012a, 2016; Rosenfield et al. 2019). For example, vocal parameters have been linked to mating and reproductive success (e.g., Apicella et al. 2007; Leongómez et al. 2014; Rosenfield et al. 2019), hunting reputation (Smith et al. 2017), and corporate hierarchy (Mayew et al. 2013). More precisely, voice pitch, the perceptual correlate of fundamental frequency (F0), has repeatedly been found to play a key role in social evaluations of male and female voices (e.g., Aung and Puts 2019; Feinberg et al. 2005; Vukovic et al. 2011): Across sexes, deeper voice pitch is relatively consistently perceived as more dominant (e.g., Borkowska and Pawlowski 2011; Ponsot et al. 2018; Vukovic et al. 2011). In contrast, deeper voice pitch is found to be more attractive in male (e.g., Feinberg et al. 2005; Puts et al. 2016), but less attractive in female speakers (e.g., Borkowska and Pawlowski 2011; Jones et al. 2010; Kandrik et al. 2016). Next to social evaluations, voice pitch has also been linked to speakers' actual characteristics, especially in males. That is, speakers with low voice pitch have higher testosterone levels (see Aung and Puts 2019 for a meta-analysis), are stronger (see Aung and Puts 2019 for a meta-analysis), and taller (see Pisanski et al. 2014 for a meta-analysis). However, note that these effects are small (see Aung and Puts 2019; Feinberg et al. 2019, 2018; Puts and Aung, 2019 for discussions).

Besides attractiveness and dominance perceptions, researchers have also started to investigate how voice pitch is related to trustworthiness in reproductive contexts. Herein, male speakers with lower voice pitch are perceived as less sexually faithful (O'Connor et al. 2011, 2014; O'Connor and Barclay 2017; Schild et al. 2020). Thus, male voices that are perceived as more attractive and dominant are at the same time perceived as belonging to speakers who are more likely to act sexually unfaithful. Because one can assume that attractive and dominant individuals are successful in both inter- and intra-sexual competition, it makes intuitively more sense that these individuals also have access to a larger pool of potential mates and are thus provided with more opportunities to be sexually unfaithful. However, evidence for an actual relation between male speakers' F0 and their infidelity is sparse. In fact, a recent study suggests that speakers can be correctly identified as cheaters regardless of their voice pitch (Hughes and Harrison 2017). In a different study, though, exploratory analyses of 181 males showed that voice pitch negatively predicted whether a speaker reported ever having been sexually unfaithful in a committed relationship. At the same time, female raters in particular also perceived speakers with low voice pitch to be less sexually faithful and made accurate judgements of who had ever cheated and who had not (Schild et al. 2020). These findings are in line with the research described above and suggest that women could use F0 as a cue to infidelity in order to avoid high fitness costs caused by infidelity, such as the loss of protection, provisioning, and investment (Geary et al. 2004; O'Connor et al. 2011). However, the study (Schild et al. 2020) also comes with certain limitations, such that only 25 males in the mostly student sample had actually stated to have ever cheated in a relationship before, which leads to relatively low statistical power. The authors also reported their findings as exploratory and explicitly urged researchers to replicate these findings. Further, the study only included male speakers, which leaves open whether F0 is also a valid indicator of 
infidelity in females. In female speakers, however, the relation between infidelity perceptions and F0 is currently unclear, with one study suggesting that higher F0 is perceived as more unfaithful (O'Connor et al. 2011) and another study suggesting no relation between F0 and infidelity perceptions (O'Connor and Barclay 2017). Additionally, no study has investigated the relation between F0 and actual infidelity in females.

Given the research gaps outlined above, we reanalyzed data of three previous studies $(N=865 ; 63,33 \%$ female; Asendorpf et al. 2011; Penke and Asendorpf 2008; Stern et al. 2020). This will not only allow us to replicate the findings by Schild et al. (2020), but also to further extend the findings to female speakers from an exploratory perspective.

\section{Hypothesis}

1. We expect that F0 negatively predicts self-reported infidelity in male speakers.

We refrained from formulating hypotheses for female speakers because evidence on infidelity perceptions in females is mixed. Further, social evaluations of attractiveness and dominance would suggest effects in opposing directions, with higher F0 being perceived as more attractive but less dominant.

\section{Planned Investigation}

We reanalyzed datasets collected for studies by Penke and Asendorpf (2008, Study 2; Dataset 1), Asendorpf et al. (2011; Dataset 2), as well as Stern et al. (2020; Dataset 3).

\section{Methods}

\section{Participants}

A total of 931 participants ( $n=331$ men, $n=600$ women; aged 18 to 54 years) were recruited in three different, independent previous studies focusing on other research questions. Sample characteristics were as follows:

Dataset 1 (Penke and Asendorpf 2008; Study 2)

Two-hundred-eighty-four participants ( $n=141$ male, $n=143$ female), aged 20 to 30 years, were recruited for a study on love, sexuality, and personality. All participants were German. Sixty percent were students.

Dataset 2 (Asendorpf et al. 2011)

Three-hundred-eighty-two participants ( $n=190$ male, $n=192$ female), aged 18 to 54 years, were recruited for the Berlin Speed Dating Study. All participants were German. Fourty-one percent reported having a university degree.

Dataset 3 (Stern et al. 2020) 
Two-hundred-sixty-six female participants, aged 18 to 35 years, were recruited for a study on psychological changes across women's ovulatory cycle. All participants were German. 97\% were students.

\section{Measures}

\section{Voice Measurement}

In all datasets, speakers provided multiple voice recordings. In detail, speakers provided three recordings (counting, free speech, reading newspaper headlines aloud) in Dataset 1, two recordings (counting, self-introduction) in Dataset 2, and three recordings $^{1}$ (free speech, vowels, German version of the rainbow passage; see Schild et al. 2020) in Dataset 3. All recordings were cut such that only the speaker is audible and then analyzed for mean F0, min F0, max F0, and F0 CV using PRAAT following the same procedure as in Schild et al. (2020). Measures of min F0, max F0, and F0 CV are used for exploratory analyses. Measurements are aggregated across recordings for each speaker. Note, however, that these parameters tend to be (highly) correlated across recordings even with different content (e.g., Mahrholz et al. 2018; Schild et al. 2020).

\section{Self-reported Infidelity}

A self-report item indicating extra-pair copulations (Datasets 1 \& 2: "With how many people did you have sex while being in a committed relationship with someone else?"; Dataset 3: "Have you ever been sexually unfaithful in an exclusive, committed relationship?") was used to create a binary indicator of infidelity. That is, individuals that report any number higher than 0 on the self-report item were coded as 1 , while individuals that report 0 were coded as 0 .

\section{Total Time in Relationships}

It has further been assessed whether and how long the participants have been in committed relationships. This variable serves as a control variable for Datasets 2 and 3 , because the longer participants have been in committed relationships, the more time they had to cheat. For these control analyses, we analyze Datasets 2 and 3 separately, as the items with which total time in relationships had been assessed differ significantly between the datasets. In Dataset 1, participants were asked to indicate their "number of relationships that lasted longer than one month" and the length of their current relationship. This variable is only used to exclude participants who have never been in a relationship so far. In Dataset 2, participants' total time spent in relationships was assessed. Participants total time spent in relationships was assessed via a categorical question with " $0=$ none, $1=<$ half a year, $2=$ about a year, $3=$ about 2 years, $4=3-5$ years, $5=$ about $1 / 4$ of my lifetime, $6=$ about half of my lifetime, $7=>$ half my lifetime". In Dataset 3, participants were asked "how many months and years in total have you

\footnotetext{
1 These recordings were done up to four times in up to four different testing sessions. However, in the current study we will only analyze the three recordings from the first session per participant.
} 
spend in exclusive, committed relationships? Please give an estimate if you are uncertain" and then entered a number for years and for months, from which a total sum score (in months) will be computed.

\section{Age}

In all three datasets, participants were asked to report their age (in years), which serves as another control variable in the robustness checks (as older participants may have had more opportunities to cheat, as they might have been in more and/or longer committed relationships in total).

\section{Attractiveness Ratings}

Voice attractiveness ratings did already exist for Datasets 1 and 2. For Dataset 1, voice recordings (counting from 1 to 10) were rated on a seven-point Likert scale from 1 "not attractive at all" to 7 "very attractive" by six trained research assistants and ratings were aggregated afterwards $(\alpha=0.80)$. For Dataset 2 , voice recordings were rated on attractiveness on a seven-point Likert scale from 1 "not attractive at all" to 7 "very attractive" by 28 (male voices) and 22 (female voices) heterosexual raters of the opposite sex, respectively. Interrater reliabilities were good $\left(\alpha_{S}>0.89\right)$, thus, ratings were aggregated (for details see Asendorpf et al. 2011). These ratings will be used for exploratory analyses (see below).

\section{Analyses}

All analyses were computed with the statistical software R (R Core Team 2016), the psych R package (Revelle 2020), and the lavaan R Package (Rosseel 2012). The analysis code is publicly available on the Open Science Framework (OSF, https://osf. io/kh7ur/). We ran separate analyses for male and female speakers. For our main analyses, we conducted a logistic regression for each dataset with mean F0 as predictor and self-reported infidelity as outcome. Two-tailed tests and $p$-values will be used to make statistical inferences. If $p$ is smaller than 0.05 , we reject the null hypothesis. In addition, we ran robustness checks controlling for total time in relationships and age, separately for each dataset due to the different operationalization of total time in relationships. Given a relation between mean F0 and self-reported infidelity in our main analyses, we conducted exploratory mediation analyses, using Datasets 1 and 2 , which test whether the relation between mean F0 and self-reported infidelity is mediated by attractiveness ratings. We also ran exploratory linear regressions for Datasets 1 and 2, using the respective continuous outcome variable (i.e., the number of extra-pair partners in Dataset $1 \& 2$ ). As in Schild et al. (2020) we also conducted exploratory logistic regression including min F0, max F0 and F0 CV as predictors of self-reported infidelity. While mean F0 represents the average voice pitch, F0 min and F0 max represent lower and upper ranges of voice pitch. F0 CV measures pitch variability which is perceived as monotonicity (see Pisanski et al. 2018; Schild et al. 2020). Lastly, we conducted an internal meta-analysis including all three datasets (and data of Schild et al. 2020). Data, script and the stage one protocol of this Registered 
Report are publicly available on the OSF (https://osf.io/kh7ur/). None of the analyses were undertaken until that protocol had received in-principal acceptance.

\section{Results}

\section{Preliminary Analyses}

We excluded $n=65$ participants from the analyses ( $n=3$ in Dataset $1, n=19$ in Dataset 2 , and $n=45$ in Dataset 3) because they reported that they have never been in a committed relationship so far and consequently did not have the chance to cheat in a committed relationship. Thus, the analyses were based on $N=281\left(N_{\text {male }}=139, N_{\text {female }}\right.$ = 142), $N=363\left(N_{\text {male }}=176, N_{\text {female }}=187\right)$, and $N=221\left(N_{\text {female }}=221\right)$, for Dataset 1 , Dataset 2, and Dataset 3, respectively. Across recordings, measures of mean F0 were correlated $(r s>0.62)$ for both men and women in all datasets. Relevant descriptive statistics are shown in Table 1.

\section{F0 and Self-reported Infidelity in Men}

\section{Dataset 1}

A logistic regression showed that mean F0 was not a significant predictor of selfreported infidelity $(O R=0.94 ; 95 \% C I:[0.66,1.33] ; p=.727)$. In an additional logistic regression neither mean F0 $(O R=0.92 ; 95 \% C I:[0.65,1.32] ; p=.658)$, nor age $(O R=$ $1.15 ; 95 \% C I:[0.81,1.63] ; p=.443)$ significantly predicted self-reported infidelity. Further, in a linear regression mean F0 did not significantly predict the number of extrapair partners $(\beta=0.05 ; 95 \% C I:[-0.12,0.22] ; p=.547)$.

\section{Dataset 2}

A logistic regression showed that mean $\mathrm{F} 0$ was a significant predictor of self-reported infidelity $(O R=0.66 ; 95 \% C I:[0.47,0.94] ; p=.020)$. In an additional logistic regression mean F0 $(O R=0.66 ; 95 \% C I:[0.45,0.97] ; p=.033)$, relationship length $(O R=$ 2.59; 95\% CI: $[1.60,4.18] ; p<.001)$, but not age $(O R=1.12 ; 95 \% C I$ : $[0.77,1.65]$; $p=.546)$ predicted self-reported infidelity significantly. These results suggest that men with lower mean F0 and a longer overall relationship length are more likely to report infidelity, but controlling for neither overall relationship length nor age reduces the predictive effect of F0 on infidelity. Further, in a linear regression, mean F0 did significantly predict the number of extra-pair partners $(\beta=-0.17 ; 95 \% C I$ : $[-0.32$, $0.03] ; p=.022)^{2}$, suggesting that men with lower F0 reported a higher number of extrapair partners.

\footnotetext{
${ }^{2}$ We removed two outliers that reported 60 and 180 extra-pair partners (remaining sample: $M=0.91, S D=$ 1.97).
} 


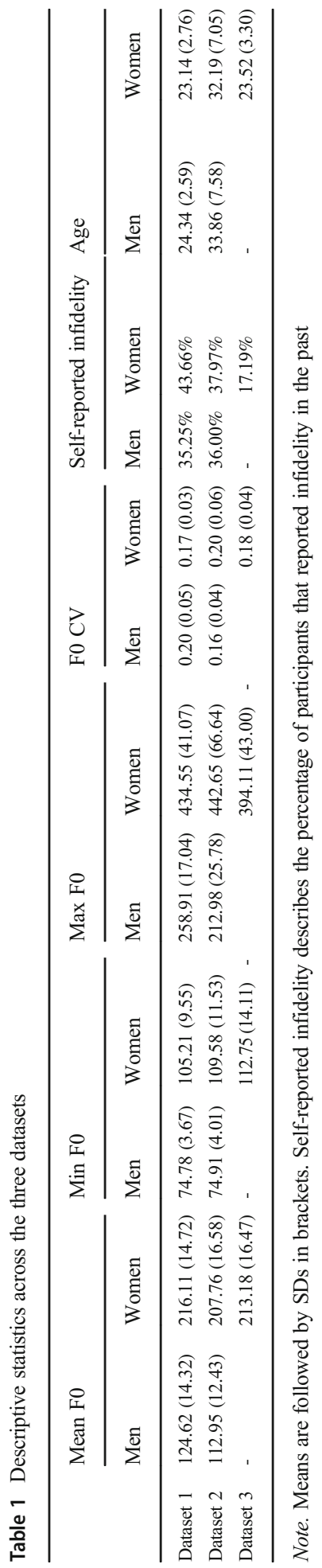




\section{Meta-analysis}

We conducted a meta-analysis using Dataset 1, Dataset 2, and the data used in Schild et al. (2020). Heterogeneity across datasets was non-significant as indicated by the Hedges estimator $(Q=4.10, d f=2, p=.129)$, and the $I 2$ statistic indicated that $51.19 \%$ of the variability was due to heterogeneity rather than chance. A random-effects metaanalysis indicated that the relation between mean F0 and self-reported infidelity was significant $(O R=0.71,95 \% C I[0.51,0.98], p=.040 ; k=3)$ as illustrated in Fig. 1. Overall, this suggests that men with lower mean F0 are more likely to report infidelity.

\section{F0 and Self-reported Infidelity in Women}

\section{Dataset 1}

A logistic regression showed that mean F0 was not a significant predictor of selfreported infidelity $(O R=0.77 ; 95 \% C I$ : $[0.55,1.09] ; p=.139)$. In an additional logistic regression neither mean F0 $(O R=0.80 ; 95 \% C I$ : $[0.56,1.15] ; p=.225)$, nor age $(O R=$ $1.12 ; 95 \% C I:[0.78,1.59] ; p=.609)$ significantly predicted self-reported infidelity. Further, in a linear regression, mean F0 did not significantly predict the number of extra-pair partners $(\beta=-0.04 ; 95 \% C I$ : $[-0.20,-0.13] ; p=.660) .^{3}$

\section{Dataset 2}

A logistic regression showed that mean F0 was a significant predictor of self-reported infidelity $(O R=0.61 ; 95 \% C I:[0.45,0.84] ; p=.002)$. In an additional logistic regression mean F0 $(O R=0.65 ; 95 \% C I:[0.45,0.94] ; p=.025)$, relationship length $(O R=$ $1.49 ; 95 \% C I:[1.02,2.18] ; p=.040)$, but not age $(O R=1.08 ; 95 \%$ CI: $[0.73,1.62]$;

\section{Study}

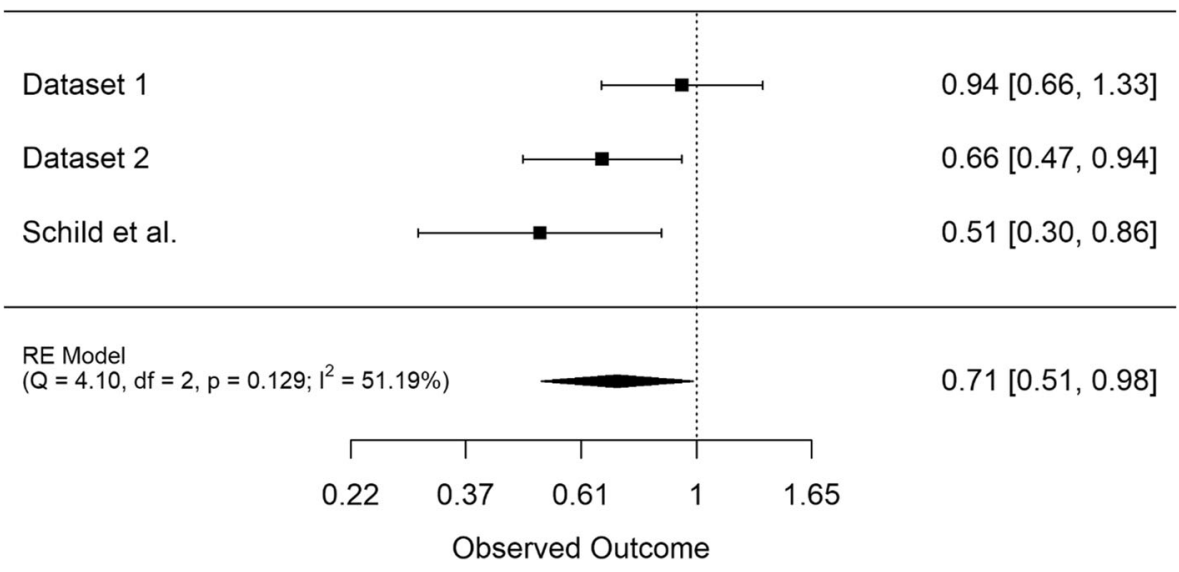

Estimate $[95 \% \mathrm{Cl}]$

Fig. 1 Random effects meta-analysis (Forest Plot) of the relation between mean F0 and self-reported infidelity in men 
$p=.696)$ significantly predicted self-reported infidelity. These results suggest that women with lower mean F0 and a longer overall relationship length are more likely to report infidelity, but controlling for neither overall relationship length nor age reduces the predictive effect of F0 on infidelity. Further, in a linear regression, mean F0 did significantly predict the number of extra-pair partners $(\beta=-0.22 ; 95 \% \mathrm{CI}$ : [$0.36,-0.07] ; p=.003$ ), suggesting that females with lower F0 reported a higher number of extra-pair partners.

\section{Dataset 3}

A logistic regression showed that mean F0 was not a significant predictor of selfreported infidelity $(O R=0.94 ; 95 \% C I:[0.66,1.33] ; p=.723)$. In an additional logistic regression neither mean $\mathrm{F} 0(O R=0.94 ; 95 \% C I:[0.64,1.39] ; p=.766)$, nor age $(O R=$ $0.99 ; 95 \% C I:[0.63,1.56] ; p=.979)$, but relationship length $(O R=2.39 ; 95 \% C I$ : $[1.53,3.73] ; p<.001)$ predicted self-reported infidelity significantly. These results suggest that females with a longer overall relationship length are more likely to report infidelity.

\section{Meta-analysis}

We conducted a meta-analysis using Dataset 1, Dataset 2, and Dataset 3. Heterogeneity across datasets was non-significant as indicated by the Hedges estimator $(Q=3.16$, $d f=2, p=.206$ ), and the $I 2$ statistic indicated that $37.19 \%$ of the variability was due to heterogeneity rather than chance. A random-effects meta-analysis indicated that the relation between mean F0 and self-reported infidelity was significant $(O R=0.76,95 \%$ CIs [0.59, 0.97], $p=.025 ; k=3$ ) as illustrated in Fig. 2. Overall, this suggests that women with lower mean F0 are more likely to report infidelity.

\section{Mediation Analyses Including Vocal Attractiveness}

In line with our protocol and given that the meta-analyses suggest significant links between mean F0 and self-reported infidelity for both sexes, we ran mediation analyses for men and women in Dataset 2 to analyze whether effects are mediated by vocal attractiveness (though not in Dataset 1, where the F0-infidelity association was not significant for either sex).

In men, mean F0 was significantly linked to self-reported infidelity $(O R=0.66 ; 95 \%$ $C I$ : $[0.47,0.94] ; p=.020)$. Although mean F0 was also significantly linked to vocal attractiveness $(r=-.58 ; 95 \% C I:[-0.67,-0.47] ; p<.001)$, the relation between mean F0 and self-reported infidelity was not mediated by vocal attractiveness: The indirect pathway of the relation between mean F0 and self-reported infidelity via vocal attractiveness was not significant $(O R=0.99 ; 95 \% C I:[0.80,1.26] ; p=.993)$. The direct pathway was significant $(O R=0.60 ; 95 \% C I$ : $[0.42,0.86] ; p=.006)$.

In women, mean F0 was significantly linked to self-reported infidelity $(O R=0.61$; 95\% CI: $[0.45,0.84] ; p=.002)$. Mean F0 was also significantly linked to vocal

\footnotetext{
3 We removed an outlier that reported 31 extra-pair partners (remaining sample: $\mathrm{M}=0.82, \mathrm{SD}=1.18$ ).
} 


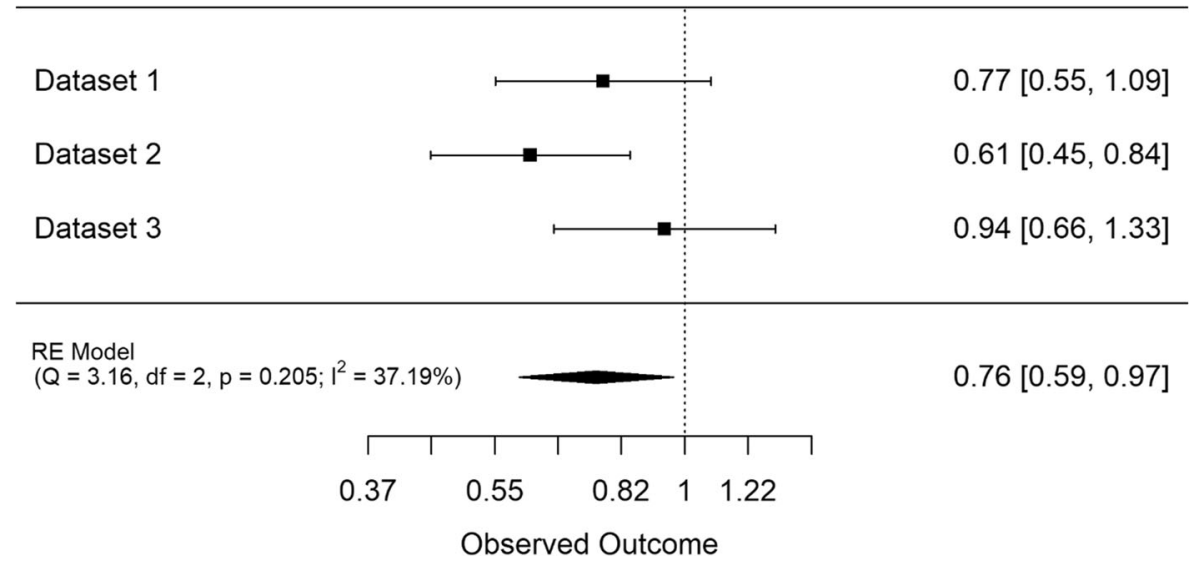

Fig. 2 Random effects meta-analysis (Forest Plot) of the relation between mean F0 and self-reported infidelity in women

attractiveness $(r=.34 ; 95 \% C I:[0.20,0.46] ; p<.001)$, and the relation between mean F0 and self-reported infidelity was partially mediated by vocal attractiveness: The indirect pathway of the relation between mean $\mathrm{F} 0$ and self-reported infidelity via vocal attractiveness was significant $(O R=0.88 ; 95 \% C I:[0.78,1.99] ; p=.037)$. Further, the direct pathway was significant $(O R=0.62 ; 95 \% C I:[0.44,0.85] ; p=.003)$. Thus, lower mean F0 predicted lower vocal attractiveness, which in turn predicted a higher likelihood of self-reported infidelity.

\section{Min F0, Max F0, FO CV, and Self-reported Infidelity}

We conducted additional exploratory logistic regression analyses investigating the relationship of self-reported infidelity with the vocal parameters Min F0, Max F0, F0 $\mathrm{CV}$, and mean F0. None of these vocal predictor variables showed a significant relation with self-reported infidelity. Details can be found in the OSF (https://osf.io/kh7ur/).

\section{Discussion}

In this Registered Report, we reanalyzed three datasets to test a potential relation between F0 and self-reported infidelity in $n=319$ male and $n=551$ female speakers. While a significant negative link between mean F0 and self-reported infidelity was found in only one out of two datasets for men and only one out of three datasets for women, two meta-analyses (accounting for the sample sizes and including the original Schild et al. 2020, data for men) suggest that lower mean F0 might be a valid indicator of higher probability of self-reported infidelity in both men and women. The one dataset that yielded significant associations for both men and women and had vocal attractiveness ratings suggests that this effect was not mediated by vocal attractiveness in men, but partially mediated by vocal attractiveness in women, such that lower mean 
F0 predicted lower vocal attractiveness, which in turn predicted a higher likelihood of self-reported infidelity. Further, where it was possible to test, relationship length was associated with higher self-reported infidelity such that participants were more likely to report extra-pair copulations in longer relationships. This is in line with the finding that sociosexual desire tends to become more unrestricted and sexual interests broaden to people outside of committed relationships after about 4 years of relationship duration, sometimes called the "4 year itch" (Fisher 1987; Penke and Asendorpf 2008). However, the effect of mean F0 on infidelity is independent of relationship length. Participants' age seemed to be unrelated to their self-reported infidelity.

\section{Why is Fo Associated With Unfaithfulness in Committed Relationships?}

Whereas previous studies report that male speakers with lower pitched voices are perceived as more likely to act sexually unfaithful in a committed relationship than speakers with higher pitched voices (O'Connor et al. 2011; O'Connor and Barclay 2017), only one previous study investigated whether mean F0 is actually linked to a higher likelihood of self-reported infidelity (Schild et al. 2020). In an exploratory finding, Schild and colleagues (Schild et al. 2020) report that men with lower F0 were, indeed, more likely to cheat in committed relationships. Further, the relation between F0 and sexual infidelity in women has not been tested so far. The current study presents evidence that F0 is actually linked to sexual unfaithfulness in men and women. Although the evidence is rather mixed in all of the separately analyzed datasets, the conducted meta-analyses suggest that men and women with lower F0 more often report to cheat in committed relationships. However, in line with the mixed findings, we recommend future research to investigate the robustness of our findings.

That mean F0 might be a valid cue to one's sexual infidelity could also explain why listeners were found to make accurate judgements about the sexual infidelity of speakers in two prior studies (Hughes and Harrison 2017; Schild et al. 2020). Picking up on a valid cue to potential infidelity might be especially relevant to avoid high fitness costs such as the loss of protection and provisioning (Geary et al. 2004) as well as parental and relationship investment (O’Connor et al. 2011). However, while no other vocal parameters in this study were found to be valid indicators of self-reported infidelity, future research should set out to investigate whether other aspects of vocal communication, such as clarity of speech (Kempe et al. 2013), are valid cues to one's infidelity.

Our findings are in line with previous findings indicating that men with lower mean F0 also report higher mating success (e.g., Puts 2005) and a higher number of sexual partners (e.g., Hughes et al. 2004), which is indicative of a less restricted sociosexual orientation. In turn, an unrestricted sociosexual orientation is linked to less commitment to romantic relationships and higher likelihoods of infidelity (Mattingly et al. 2011; Penke and Asendorpf 2008). But why is F0 associated with a higher likelihood of infidelity? Romantic infidelity can be the result of situational (e.g. opportunities) and dispositional factors (Blow and Hartnett 2005; Hilbig et al. 2015). With regard to opportunities for infidelity, lower mean F0 in men is associated with both perceptions of attractiveness and dominance (e.g., Puts et al. 2016), so it increases success in both being chosen by the opposite sex and intrasexual competition. The association can thus not distinguish between these two routes to infidelity opportunities, though two studies 
suggest that success in male-male competition, rather than female mate choice, is a more important predictor of male number of sexual partners and that male F0 is under stronger intrasexual than intersexual selection (Hill et al. 2013; Kordsmeyer et al. 2018). In contrast, lower female mean F0 is perceived as more dominant but less attractive (e.g., Borkowska and Pawlowski 2011; Jones et al. 2010). Interestingly lower, not higher, mean F0 predicted infidelity in women. This could either mean that being perceived as dominant is important for female infidelity opportunities, just as it is for men. Alternatively, it could be interpreted as less vocally attractive women being more likely to be romantically unfaithful, which is corroborated by the partial mediation of the F0infidelity association by lower rated vocal attractiveness in Dataset 2. Vocal attractiveness contributes to women's likelihood of being chosen by potential mates over and beyond physical attractiveness (Asendorpf et al. 2011). Thus, it might be that less vocally attractive women end up with less opportunity to engage in a committed relationship with a preferred partner on a competitive mating market with mutual mate choice, as is typical for modern humans (Penke et al. 2008). If this is the case, these women might use infidelity as a mate switching strategy (Buss et al. 2017). As another alternative, a lower F0 and the disposition for infidelity might share a common cause in both men and women. A candidate would be androgenic masculinization throughout development. Both, mean F0 (Puts et al. 2012a, b) and unrestricted sociosexual desire (Penke and Asendorpf 2008; Schmitt 2005), as well as the closely related desire for sexual variety (Schmitt and International Sexuality Description Project 2003), are strongly sexually dimorphic in humans. Importantly, higher masculinity is also linked to less restricted sociosexual orientation (Ostovich and Sabini 2004) and more sexual partners across the lifespan (Burri et al. 2015) in women, potentially explaining our findings. Lastly, given that women lower their mean F0 when talking to more attractive men (Hughes et al. 2010), when speaking to men they prefer (Pisanski et al. 2018) and when trying to sound sexy or attractive (Hughes et al. 2014), it might be that lower mean F0 indicates general interest and attracts more opportunities for infidelity. Importantly, all these potential explanations are not mutually exclusive, and might thus be addressed explicitly by future research.

\section{Limitations}

Our investigation has four potential limitations in particular. First, due to the item wording, our infidelity measure was only a proxy of self-reported infidelity in Datasets 1 and 2: While one can assume that a majority of extra-pair copulations are, indeed, best described by acts of infidelity, other extra-pair copulations might actually be accepted by the partner (e.g., in polyamorous couples or open relationships, which were not assessed). Thus, our outcome measure might contain noise. However, note that only around 5\% of relationships in western countries (such as those in which our data were collected) are consensually non-monogamous (Rubin et al. 2014). Second, as in Schild et al. (2020), we were only able to analyze whether individuals have ever cheated on any of their partners. We are not able to investigate or draw any conclusions about (a) how many of their partners they have cheated on (just one, all of them, or anything in between), (b) what were the reasons for cheating, and (c) whether cheating that does not involve sexual intercourse (e.g., kissing) is also related to F0. Third, for assessing infidelity, we relied on self-report measures. However, as infidelity in 
committed relationships is rather socially undesirable (Mogilski et al. 2014), there is a chance that not all participants gave honest responses to these questions, although all surveys were administered completely anonymous. Fourth, although the overall sample size of this investigation was relatively large, the asymmetric distribution of cheaters and non-cheaters decreased the statistical power of this investigation. In detail, 39\%, $37 \%$, and $17 \%$ of the study participants reported infidelity in Dataset 1, Dataset 2, and Dataset 3, respectively. We strongly encourage future studies to replicate our study and resolve potential problems that limit the interpretability of the current study's findings.

\section{Conclusions}

A significant negative link between mean F0 and self-reported infidelity was found in only one out of two datasets for men and only one out of three for women. However, two meta-analyses (including data from Schild et al. 2020) find that lower mean F0 is indicative of a higher likelihood of self-reported infidelity in men as well as women. While these findings are in line with prior research that suggests that more masculine traits, such as a deep voice, are indicative of sociosexual orientation and, in turn, infidelity, future studies should set out to further extend these findings.

Funding Open Access funding enabled and organized by Projekt DEAL.

\section{Compliance with Ethical Standards}

Conflict of Interest The authors declared that they have no conflict of interest.

Open Access This article is licensed under a Creative Commons Attribution 4.0 International License, which permits use, sharing, adaptation, distribution and reproduction in any medium or format, as long as you give appropriate credit to the original author(s) and the source, provide a link to the Creative Commons licence, and indicate if changes were made. The images or other third party material in this article are included in the article's Creative Commons licence, unless indicated otherwise in a credit line to the material. If material is not included in the article's Creative Commons licence and your intended use is not permitted by statutory regulation or exceeds the permitted use, you will need to obtain permission directly from the copyright holder. To view a copy of this licence, visit http://creativecommons.org/licenses/by/4.0/.

\section{References}

Apicella, C. L., Feinberg, D. R., \& Marlowe, F. W. (2007). Voice pitch predicts reproductive success in male hunter-gatherers. Biology Letters, 3(6), 682-684. https://doi.org/10.1098/rsb1.2007.0410.

Asendorpf, J. B., Penke, L., \& Back, M. D. (2011). From dating to mating and relating: Predictors of initial and long-term outcomes of speed-dating in a community sample. European Journal of Personality, 25(1), 16-30. https://doi.org/10.1002/per.768.

Aung, T., \& Puts, D. (2019). Voice pitch: A window into the communication of social power. Current Opinion in Psychology. https://doi.org/10.1016/j.copsyc.2019.07.028.

Blow, A. J., \& Hartnett, K. (2005). Infidelity in Committed Relati0nships Ii: A Substantive Review. Journal of Marital and Family Therapy, 31(2), 217-233. https://oi.org/10.1111/j.1752-0606.2005.tb01556.x.

Borkowska, B., \& Pawlowski, B. (2011). Female voice frequency in the context of dominance and attractiveness perception. Animal Behaviour, 82(1), 55-59. https://doi.org/10.1016/j.anbehav.2011.03.024. 
Burri, A., Spector, T., \& Rahman, Q. (2015). Common Genetic Factors among Sexual Orientation, Gender Nonconformity, and Number of Sex Partners in Female Twins: Implications for the Evolution of Homosexuality. The Journal of Sexual Medicine, 12(4), 1004-1011. https://doi.org/10.1111/jsm.12847.

Buss, D. M., Goetz, C., Duntley, J. D., Asao, K., \& Conroy-Beam, D. (2017). The mate switching hypothesis. Personality and Individual Differences, 104, 143-149. https://doi.org/10.1016/j.paid.2016.07.022.

Feinberg, D. R., Jones, B. C., \& Armstrong, M. M. (2018). Sensory Exploitation, Sexual Dimorphism, and Human Voice Pitch. Trends in Ecology \& Evolution, 33(12), 901-903. https://doi.org/10.1016/j.tree. 2018.09.007.

Feinberg, D. R., Jones, B. C., \& Armstrong, M. M. (2019). No Evidence That Men's Voice Pitch Signals Formidability. Trends in Ecology \& Evolution, 34(3), 190-192. https://doi.org/10.1016/j.tree.2018.12. 014.

Feinberg, D. R., Jones, B. C., Little, A. C., Burt, D. M., \& Perrett, D. I. (2005). Manipulations of fundamental and formant frequencies influence the attractiveness of human male voices. Animal Behaviour, 69(3), 561-568. https://doi.org/10.1016/j.anbehav.2004.06.012.

Fisher, H. (1987). The four year itch. Natural History, 10, 22-29.

Geary, D. C., Vigil, J., \& Byrd-Craven, J. (2004). Evolution of human mate choice. The Journal of Sex Research, 41(1), 27-42. https://doi.org/10.1080/00224490409552211.

Hilbig, B. E., Moshagen, M., \& Zettler, I. (2015). Truth will out: Linking personality, morality, and honesty through indirect questioning. Social Psychological and Personality Science, 6(2), 140-147. https://doi. org/10.1177/1948550614553640.

Hill, A. K., Hunt, J., Welling, L. L. M., Cárdenas, R. A., Rotella, M. A., Wheatley, J. R., Dawood, K., Shriver, M. D., \& Puts, D. A. (2013). Quantifying the strength and form of sexual selection on men's traits. Evolution and Human Behavior, 34(5), 334-341. https://doi.org/10.1016/j.evolhumbehav.2013.05.004.

Hughes, S. M., Dispenza, F., \& Gallup, G. G. (2004). Ratings of voice attractiveness predict sexual behavior and body configuration. Evolution and Human Behavior, 25(5), 295-304. https://doi.org/10.1016/j. evolhumbehav.2004.06.001.

Hughes, S. M., Farley, S. D., \& Rhodes, B. C. (2010). Vocal and physiological changes in response to the physical attractiveness of conversational partners. Journal of Nonverbal Behavior, 34(3), 155-167. https://doi.org/10.1007/s10919-010-0087-9

Hughes, S. M., \& Harrison, M. A. (2017). Your cheatin' voice will tell on you: Detection of past infidelity from voice. Evolutionary Psychology, 15(2), 1474704917711513. https://doi.org/10.1177/1474704917711513.

Hughes, S. M., Mogilski, J. K., \& Harrison, M. A. (2014). The perception and parameters of intentional voice manipulation. Journal of Nonverbal Behavior, 38(1), 107-127. https://doi.org/10.1007/s10919-013-0163$\mathrm{Z}$.

Jones, B. C., Feinberg, D. R., DeBruine, L. M., Little, A. C., \& Vukovic, J. (2010). A domain-specific opposite-sex bias in human preferences for manipulated voice pitch. Animal Behaviour, 79(1), 57-62. https://doi.org/10.1016/j.anbehav.2009.10.003.

Kandrik, M., Hahn, A. C., Wincenciak, J., Fisher, C. I., Pisanski, K., Feinberg, D. R., et al. (2016). Are men's perceptions of sexually dimorphic vocal characteristics related to their testosterone levels? PLoS One, 11(11), e0166855. https://doi.org/10.1371/journal.pone.0166855.

Kempe, V., Puts, D. A., \& Cárdenas, R. A. (2013). Masculine Men Articulate Less Clearly. Human Nature, 24(4), 461-475. https://doi.org/10.1007/s12110-013-9183-y.

Kordsmeyer, T. L., Hunt, J., Puts, D. A., Ostner, J., \& Penke, L. (2018). The relative importance of intra- and intersexual selection on human male sexually dimorphic traits. Evolution and Human Behavior, 39(4), 424 436. https://doi.org/10.1016/j.evolhumbehav.2018.03.008.

Leongómez, J. D., Binter, J., Kubicová, L., Stolařová, P., Klapilová, K., Havlíček, J., \& Roberts, S. C. (2014). Vocal modulation during courtship increases proceptivity even in naive listeners. Evolution and Human Behavior, 35(6), 489-496. https://doi.org/10.1016/j.evolhumbehav.2014.06.008.

Mahrholz, G., Belin, P., \& McAleer, P. (2018). Judgements of a speaker's personality are correlated across differing content and stimulus type. PLoS One, 13(10), e0204991. https://doi.org/10.1371/journal.pone. 0204991 .

Mattingly, B. A., Clark, E. M., Weidler, D. J., Bullock, M., Hackathorn, J., \& Blankmeyer, K. (2011). Sociosexual orientation, commitment, and infidelity: A mediation analysis. The Journal of Social Psychology, 151(3), 222-226. https://doi.org/10.1080/00224540903536162.

Mayew, W. J., Parsons, C. A., \& Venkatachalam, M. (2013). Voice pitch and the labor market success of male chief executive officers. Evolution and Human Behavior, 34(4), 243-248. https://doi.org/10.1016/j. evolhumbehav.2013.03.001. 
Mogilski, J. K., Wade, T. J., \& Welling, L. L. M. (2014). Prioritization of potential mates' history of sexual fidelity during a conjoint ranking task. Personality and Social Psychology Bulletin, 40(7), 884-897. https://doi.org/10.1177/0146167214529798.

O'Connor, J. J. M., \& Barclay, P. (2017). The influence of voice pitch on perceptions of trustworthiness across social contexts. Evolution and Human Behavior, 38(4), 506-512. https://doi.org/10.1016/j. evolhumbehav.2017.03.001.

O’Connor, J. J. M., Pisanski, K., Tigue, C. C., Fraccaro, P. J., \& Feinberg, D. R. (2014). Perceptions of infidelity risk predict women's preferences for low male voice pitch in short-term over long-term relationship contexts. Personality and Individual Differences, 56, 73-77. https://doi.org/10.1016/j.paid. 2013.08.029.

O’Connor, J. J. M., Re, D. E., \& Feinberg, D. R. (2011). Voice pitch influences perceptions of sexual infidelity. Evolutionary Psychology, 9(1), 147470491100900100. https://doi.org/10.1177/147470491100900109.

Ostovich, J. M., \& Sabini, J. (2004). How are sociosexuality, sex drive, and lifetime number of sexual partners related? Personality and Social Psychology Bulletin, 30(10), 1255-1266. https://doi.org/10.1177/ 0146167204264754.

Penke, L., \& Asendorpf, J. B. (2008). Beyond global sociosexual orientations: A more differentiated look at sociosexuality and its effects on courtship and romantic relationships. Journal of Personality and Social Psychology, 95(5), 1113-1135. https://doi.org/10.1037/0022-3514.95.5.1113.

Penke, L., Todd, P. M., Lenton, A. P., \& Fasolo, B. (2008). How self-assessments can guide human mating decisions. In Mating intelligence: Sex, relationships, and the mind's reproductive system (pp. 37-75). Mahwah: Lawrence Erlbaum Associates Publishers.

Pisanski, K., Fraccaro, P. J., Tigue, C. C., O’Connor, J. J. M., Röder, S., Andrews, P. W., Fink, B., DeBruine, L. M., Jones, B. C., \& Feinberg, D. R. (2014). Vocal indicators of body size in men and women: A metaanalysis. Animal Behaviour, 95, 89-99. https://doi.org/10.1016/j.anbehav.2014.06.011.

Pisanski, K., Oleszkiewicz, A., Plachetka, J., Gmiterek, M., \& Reby, D. (2018). Voice pitch modulation in human mate choice. Proceedings of the Royal Society B: Biological Sciences, 285(1893), 20181634. https://doi.org/10.1098/rspb.2018.1634.

Ponsot, E., Burred, J. J., Belin, P., \& Aucouturier, J.-J. (2018). Cracking the social code of speech prosody using reverse correlation. Proceedings of the National Academy of Sciences, 115(15), 3972-3977. https:// doi.org/10.1073/pnas.1716090115.

Puts, D.A. (2005). Mating context and menstrual phase affect women's preferences for male voice pitch. Evolution and Human Behavior, 26(5), 388-397. https://doi.org/10.1016/j.evolhumbehav.2005.03.001.

Puts, D. A., Apicella, C. L., \& Cárdenas, R. A. (2012). Masculine voices signal men's threat potential in forager and industrial societies. Proceedings of the Royal Society B: Biological Sciences, 279(1728), 601609. https://doi.org/10.1098/rspb.2011.0829.

Puts, D. A., \& Aung, T. (2019). Does men's voice pitch signal formidability? A reply to Feinberg et al. Trends in Ecology \& Evolution, 34(3), 189-190. https://doi.org/10.1016/j.tree.2018.12.004.

Puts, D. A., Hill, A. K., Bailey, D. H., Walker, R. S., Rendall, D., Wheatley, J. R., et al. (2016). Sexual selection on male vocal fundamental frequency in humans and other anthropoids. Proceedings of the Royal Society B, 283(1829), 20152830. https://doi.org/10.1098/rspb.2015.2830.

Puts, D. A., Jones, B. C., \& DeBruine, L. M. (2012). Sexual selection on human faces and voices. The Journal of Sex Research, 49(2-3), 227-243. https://doi.org/10.1080/00224499.2012.658924.

R Core Team. (2016). R: a language and environment for statistical computing. R Foundation for Statistical Computing, Vienna, Austria. Retrieved from http://www.R-project.org/.

Revelle., W. (2020). Psych: procedures for psychological, psychometric, and personality research.

Rosenfield, K. A., Sorokowska, A., Sorokowski, P., \& Puts, D. A. (2019). Sexual selection for low male voice pitch among Amazonian forager-horticulturists. Evolution and Human Behavior. https://doi.org/10.1016/ j.evolhumbehav.2019.07.002.

Rosseel., Y. (2012). lavaan: an R package for structural equation modeling. Journal of Statistical Software, 48(2), 1-36.

Rubin, J., Moors, A., Matsick, J., Ziegler, A., \& Conley, T. (2014). On the margins: Considering diversity among consensually non-monogamous relationships. Psychology Faculty Articles and Research. https:// digitalcommons.chapman.edu/psychology_articles/133.

Schild, C., Stern, J., \& Zettler, I. (2020). Linking men's voice pitch to actual and perceived trustworthiness across domains. Behavioral Ecology. https://doi.org/10.1093/beheco/arz173.

Schmitt, D.P., \& International Sexuality Description Project. (2003). Universal sex differences in the desire for sexual variety: tests from 52 nations, 6 continents, and 13 islands. Journal of Personality and Social Psychology, 85(1), 85-104. https://doi.org/10.1037/0022-3514.85.1.85. 
Schmitt, D. P. (2005). Sociosexuality from Argentina to Zimbabwe: A 48-nation study of sex, culture, and strategies of human mating. The Behavioral and Brain Sciences, 28(2), 247-275; discussion 275-311. https://doi.org/10.1017/s0140525x05000051.

Smith, K. M., Olkhov, Y. M., Puts, D. A., \& Apicella, C. L. (2017). Hadza men with lower voice pitch have a better hunting reputation. Evolutionary Psychology, 15(4), 1474704917740466. https://doi.org/10.1177/ 1474704917740466.

Stern, J., Kordsmeyer, T. L., \& Penke, L. (2020). A longitudinal evaluation of ovulatory cycle shifts in women's mate attraction and preferences. Manuscript submitted for publication.

Vukovic, J., Jones, B. C., Feinberg, D. R., DeBruine, L. M., Smith, F. G., Welling, L. L. M., \& Little, A. C. (2011). Variation in perceptions of physical dominance and trustworthiness predicts individual differences in the effect of relationship context on women's preferences for masculine pitch in men's voices. British Journal of Psychology, 102(1), 37-48. https://doi.org/10.1348/000712610X498750.

Publisher's Note Springer Nature remains neutral with regard to jurisdictional claims in published maps and institutional affiliations.

\section{Affiliations}

\section{Christoph Schild ${ }^{1,2} \cdot$ Julia Stern ${ }^{3} \cdot$ Lars Penke $^{3} \cdot$ Ingo Zettler $^{1}$}

1 Department of Psychology, University of Copenhagen, Øster Farimagsgade 2a, 1353 Copenhagen, Denmark

2 Department of Psychology, University of Siegen, Adolf-Reichwein-Str. 2a, 57068 Siegen, Germany

3 Department of Psychology \& Leibniz ScienceCampus Primate Cognition, University of Goettingen, Gosslerstrasse 14, 37073 Goettingen, Germany 